\title{
Remote sensing and modeling of landslides: detection, monitoring and risk evaluation
}

\author{
Dalia Bach Kirschbaum • Hiroshi Fukuoka
}

Published online: 31 January 2012

(C) Springer-Verlag (outside the USA) 2012

Landslides are one of the most pervasive hazards in the world, resulting in more fatalities and economic damage than is generally recognized. Occurring over an extensive range of lithologies, morphologies, hydrologies, and climates, mass movements can be triggered by intense or prolonged rainfall, seismicity, freeze/thaw processes, and anthropogenic activities, among other factors. The location, size, and timing of these processes are characteristically difficult to predict and assess because of their localized spatial scales, distribution, and complex interactions between rainfall infiltration, hydromechanical properties of the soil, and the underlying surface composition. However, the increased availability, accessibility, and resolution of remote sensing data offer a new opportunity to explore issues of landslide susceptibility, hazard, and risk over a variety of spatial scales. This special issue presents a series of papers that investigate the sources, behavior, and impacts of different mass movement types using a diverse set of data sources and evaluation methodologies.

This special issue of Environmental Earth Sciences initially announced an open call for papers related to the above-mentioned topics. After the peer-review process, a selection was made of papers by researchers representing different countries on topics within the subject of remote sensing of landslide hazard and risk. The diverse nature of the landslide types, evaluation methodologies, study regions, and data sources employed in the eleven research

D. B. Kirschbaum ( $\square)$

NASA Goddard Space Flight Center, Greenbelt, MD, USA

e-mail: dalia.b.kirschbaum@nasa.gov

H. Fukuoka

Research Center on Landslides, Disaster Prevention Research Institute, Kyoto University, Gokasho, Uji,

Kyoto 611-0011, Japan papers presented here underscore the significance and impact of mass movements worldwide.

The research papers fall within several broad categories. Several papers employ statistical or deterministic methodologies to model the impact of shallow debris flows in tropical or subtropical climates at varying spatial scales. These studies used retrospective evaluations of extreme landslide-triggering precipitation events to develop and validate quantitative models. A second set of papers consider landslide and rockfall hazards in relation to estimated local soil moisture, temperature, seismicity, and rainfall information. These papers investigate the mechanisms affecting slope failure in several different regions around the world. A third category of research employed remotely sensed information to evaluate slope movement over time using Interferometric Synthetic Aperture Radar (In-SAR) data. Lastly, a fourth selection of papers investigates the contribution of anthropogenic building and development to slope instability. Through case studies these papers assess landslide risk affecting populations close to such potentially unstable areas.

As guest editors, we would like to express our appreciation to the Editorial Board of the Journal of Environmental Earth Sciences for publishing this special issue and supporting this important topic. We hope that through these series of data, methodology, and case study investigations, the articles reveal new and exciting avenues of study within the topic of remote sensing and modeling of landslides. 